\title{
ALGORITHM OF CONTROLLING REACTIVE POWER COMPENSATOR WITH NONLINEAR LOADS
}

\author{
Igor Vorotnikov, Maxim Mastepanenko, Shaliko Gabrielian, Nataliya Kasyanova \\ Stavropol State Agrarian University \\ eaim2015@mail.ru
}

\begin{abstract}
A possibility of controlling the reactive power compensator for non-sinusoidal modes is considered by using the algorithm for decomposition of instant power into components in the modes of phase shift of the current comparatively supplying voltages and distortion in the form of the current curve at real time. A comparison is made between two calculation methods for determining the compensator current that is connected in parallel to the nonlinear load. The effectiveness of the proposed calculation methodology is confirmed by the results of simulation modeling.
\end{abstract}

Key words: algorithm, instant power, forming powers, compensator, power factor.

\section{Introduction}

The development of power electronics, success in the creation of microprocessor systems and the constant tightening of the requirements for saving electricity have led to a paradoxical situation. In order to save electricity, various adjustable electric power converters are created, allowing only the necessary minimum energy to be transmitted to the actuator. But along with this, the converters consume in most cases a non-linear current, which is the source of a wide range of disturbances in electric networks, which in turn leads to a deterioration in the quality of electrical energy, increased energy losses and a decrease in the performance of electrical equipment.

Currently, insufficient attention is paid to issues of reactive power compensation in rural electric networks. This problem is most relevant for rural electric networks, where losses of electrical energy due to low power quality indicators account for up to $30 \%$ of the total losses. Also, the reactive current additionally loads the transmission lines, which leads to an increase in the cross-sections of wires and cables and, accordingly, to an increase in the capital costs for external and in-site networks; Reactive power, along with active power, is accounted for by the electricity supplier and is payable at current tariffs, and therefore accounts for a significant portion of the electricity bills.

The purpose of this work is to increase the effectiveness of informational, measuring and control systems for reactive power compensation plants for agricultural and industrial consumers operating under non-sinusoidal modes.

The purpose is achieved by using the control algorithm of the reactive power compensator. The algorithm is based on the decomposition of the instantaneous power curve into components in real time. The control algorithm is valid for the cases of lead advance or lag with respect to the voltage and the mismatch of the voltage and voltage curves of the network and the load current.

Personal computers, uninterruptible power supplies, gas-discharge lamps, servo drives, variable speed motors are non-linear loads and the current, consumed by these consumers, has an expressed impulse character and is a non-sinusoidal periodic signal, having in its composition higher harmonic components.

It should be noted that the share of these electricity consumers in the agricultural sector is steadily growing due to the intensive introduction of modern technologies for the production and processing of agricultural products.

An obvious method of increasing the efficiency of electricity transmission with established quality indicators, especially for nonlinear loads, is reactive power compensation.

It is known that the theory of reactive power is very ambiguous, and sometimes contradictory.

In the literary review of this work [1] is noted that "the beginning of the theory development of energy processes was laid in the last century by the works of M. Dolevo-Dobrovolsky, C. Steinmetz, C. Puglia and other scientists. By the end of the past century, the concepts of active, "wattless" (reactive) power and electrical resistance for circuits with sinusoidal currents and voltages were formulated. However, the actual theory of energy processes in circuits with non-sinusoidal currents 
and voltages begins to develop only after the appearance of works by Budyan (1927) and Frize (1931). Until now, scientists in many countries are working intensively in this field.

Numerous attempts have been made to generalize the concept of reactive power to non-sinusoidal processes or to introduce other general concepts that replace it. However, these efforts have not led to the creation of a generally accepted energy theory that adequately reflects the energy processes in the general case, in the presence of distortions both in current and in tension".

The organic deficiency of all frequency methods is the lack of physical justification for mathematical procedures because of the impossibility of using the concepts of instant power components. As a consequence, there are effects that indicate the inherent inconsistency of this method.

\section{Materials and methods}

In his works [2; 3], K.S. Demirchyan notes that "the extension of the method for determining the reactive power produced for sinusoidal functions to more general cases leads to incorrect results. This is particularly true for approaches related to the decomposition of nonsinusoidal functions into Fourier series or other functional series. This representation of the physical process, in addition to the sharp complication of the analysis itself, involuntarily pushes the researcher to look not so much physically justified as mathematically observable expressions. "

We recall the main factors of the calculation technique [2] from the representation of the current (voltage) in the time coordinate system in the form of two components: collinear voltage (current) and orthogonal to them in the instantaneous values. The current (voltage) vector is represented as a sum

$$
\vec{i}=\vec{i}_{U}+\bar{i}_{O R} \text { or } \vec{u}=\vec{u}_{I}+\vec{u}_{O R},
$$

where $\vec{i}_{U}, \vec{u}_{I}$ are the current (voltage) components collinear with the voltage (current) vector; $\bar{i}_{O R}, \vec{u}_{O R}$ are current (voltage) components orthogonal to the voltage (current) vector.

The collinear components of the current and voltage are determined by the following expressions

$$
\vec{i}_{U}=\vec{u}(\vec{u}, \vec{i}) /(\vec{u}, \vec{u}) ; \vec{u}_{I}=\vec{i}(\vec{u}, \vec{i}) /(\vec{i}, \vec{i}),
$$

Here the expressions in parentheses denote the operation of the scalar product of vectors whose coordinates are the values of the sample times and the instant values of the quantities corresponding to these reports.

For the instant power $\vec{p}=\vec{i} \vec{u}$, the following relations are true.

If the current curve is expanded, then:

$$
\vec{p}=\vec{u}\left(\vec{i}_{U}+\vec{i}_{O R}\right)=\vec{u} \vec{i}_{U}+\vec{u} \vec{i}_{O R}=\vec{p}_{U}+\vec{p}_{O R}=\vec{p}_{U}+\vec{p}_{q}=\vec{p}_{a}+\vec{p}_{q},
$$

And if we expand the voltage curve, we have:

$$
\vec{p}=\vec{i}\left(\vec{u}_{i}+\vec{u}_{O R}\right)=\vec{i} \vec{u}_{i}+\vec{i} \vec{u}_{O R}=\vec{p}_{i}+\vec{p}_{O R}=\vec{p}_{i}+\vec{p}_{q}=\vec{p}_{a}+\vec{p}_{q}
$$

where $\vec{p}_{U}, \vec{p}_{i}, \vec{p}_{a}$ are instantaneous power components that determine only the active power,

$\vec{p}_{q}$ is the component that adds the active to the instant.

It should be noted that the equality of the last terms in expressions (3) and (4) means the equivalence of the expansion of the power curve along the current curve by the expansion of the power curve along the voltage curve [4].

It is worthy of note that

$$
P=(\vec{u}, \vec{i}) / T \text { и } U^{2}=(\vec{u}, \vec{u}) / T,
$$

where $P$ is the active power, average value, $\mathrm{W}$;

$T$ is the period of the voltage of the industrial frequency, s;

$U$ is the effective value of the voltage, $\mathrm{V}$. 
And also that

$$
\vec{i}_{O R}=\vec{i}-\vec{i}_{U}, \vec{p}_{q}=\vec{u} \vec{i}_{O R},\left(\vec{u}, \vec{i}_{O R}\right)=\left(\vec{u},\left(\vec{i}-\vec{i}_{U}\right)=0\right.
$$

Expressions (6) determine the most informative parameters of the current and instant power, since they characterize both the exchange processes, and the distortions of the shapes of the stress and current curves, and characterize the instant generalized reactive power.

Following the principle of uniform definition of active and reactive powers, the average value of the instant generalized reactive power is defined as the arithmetic mean product of absolute instant values:

$$
Q_{O R}=\left(|\vec{u}|,\left|\vec{i}_{O R}\right|\right) / T
$$

Following the general procedure for determining the average value of a periodic function, one can derive an expression for some power, in this case both exchange and minimizate, and in the generalized sense of the reactive power that KS. Demirchyan called ortho-power:

$$
Q=(\pi / 2) Q_{O R}=(\pi / 2)\left(|\vec{u}|,\left|\vec{i}_{O R}\right|\right) / T=(\pi /(2 T)(|\vec{u}|,|\vec{i}-\vec{u}(\vec{i}, \vec{u})| /(\vec{u}, \vec{u}))
$$

or

$$
Q=(\pi /(2 T)(|\vec{u}|,|\vec{i}-\vec{u} G|),
$$

where $G$ - conductivity.

Simulation modeling of electromagnetic processes during the operation of a reactive power compensator with a control system based on the above proportions was carried out in the Matlab/Simulink environment using the virtual installation proposed in the work [5].

The installation model with the changes introduced by the authors of this article is shown in Figure 1.

The main elements of the model are a nonlinear load in the form of a controllable rectifier (Rectifier) with an active-inductive load RL and a compensator in the form of a single-phase bridge inverter (Universal Bridge) with a capacitor $\mathrm{C}$ in the DC circuit and a reactor $\mathrm{Lr}$ in the alternating current circuit.

In this case, the compensator performs the functions of the active harmonic filter, generating a current to the network, the shape of which eliminates distortions introduced into the network by the load current.

The principles of operation of such units are well known, but the ways of controlling them, that is, the algorithmization of control systems for expansion joints, continues to be very relevant.

The purpose of this article is to evaluate the efficiency of the compensating installation under consideration using the control algorithm-in terms of the fundamental harmonic of the network current adopted in the prototype, in comparison with the proposed control algorithm-the decomposition of the instant current into components with sinusoidal voltage of the network.

To solve this problem, an additional block-sum-tor (subtractor) of control signals, obtained by different compared computational methods, was introduced into the model.

Detailing of one more input by the authors in the installation of an additional block Subsystem1 is shown in Figure 2.

The upper part of the figure shows the channel for calculating the average value of active power for the period by the instant values of the input voltage and current signals. In the lower part - the channel for calculating the square of the active network.

The Divide block provides a calculation of the conductivity G. As a result, an output corresponding to the collinear component of the network current $i_{u}=u(u, i) /(u, u)$ is obtained at the output Out1 in accordance with expression (2).

The orthogonal current component is calculated in the Hist_Control block by subtracting the line current from the specified compensator control current. 


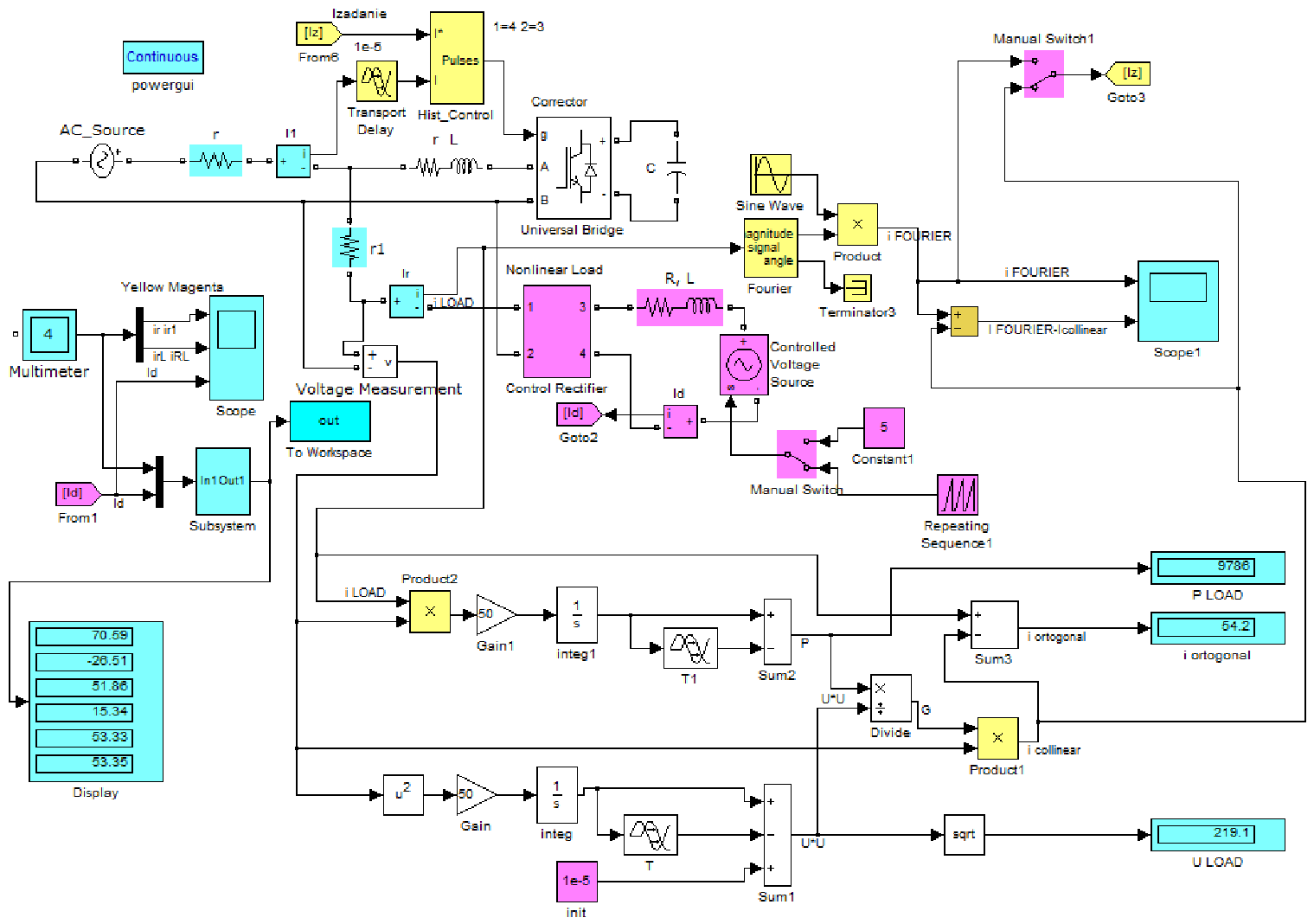

Fig. 1. Virtual installation model

The results of simulation modeling are presented in Figure 3, where the upper graph corresponds to the compensation current of the fundamental harmonic of the load current obtained by splitting the latter into a harmonic Fourier series (prototype).

The lower graph of Figure 3 corresponds to the difference in currents: the upper graph and the collinear voltage of the load current component obtained by the proposed calculation technique.

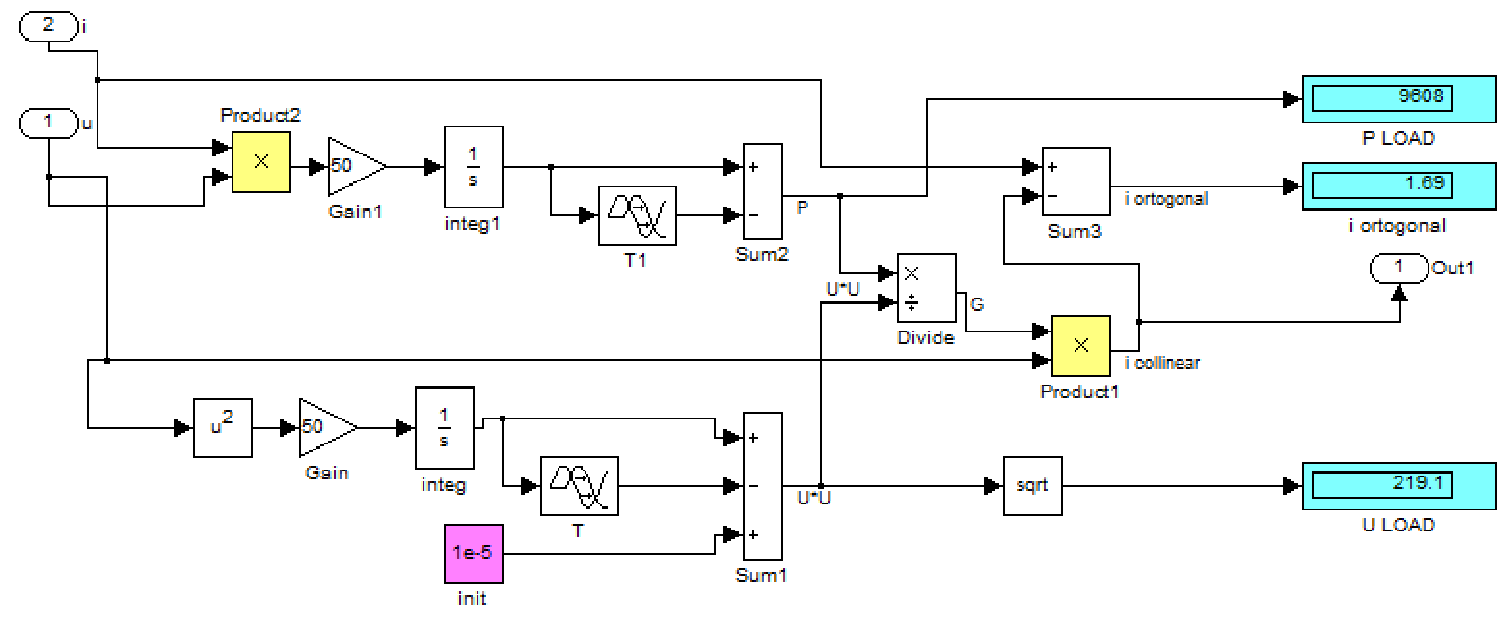

Fig. 2. Model of the block for calculating the components of the nonlinear load current: collinear and orthogonal 
The compensation current of the fundamental harmonic

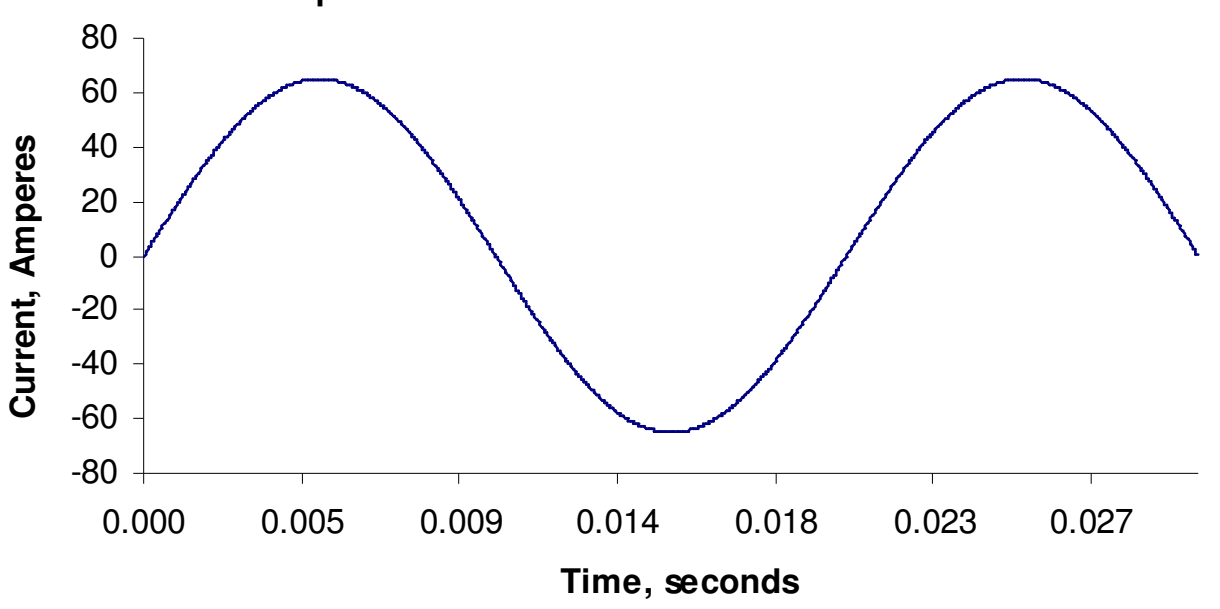

The difference in currents: the current of fudamental harmonic and the collinear current

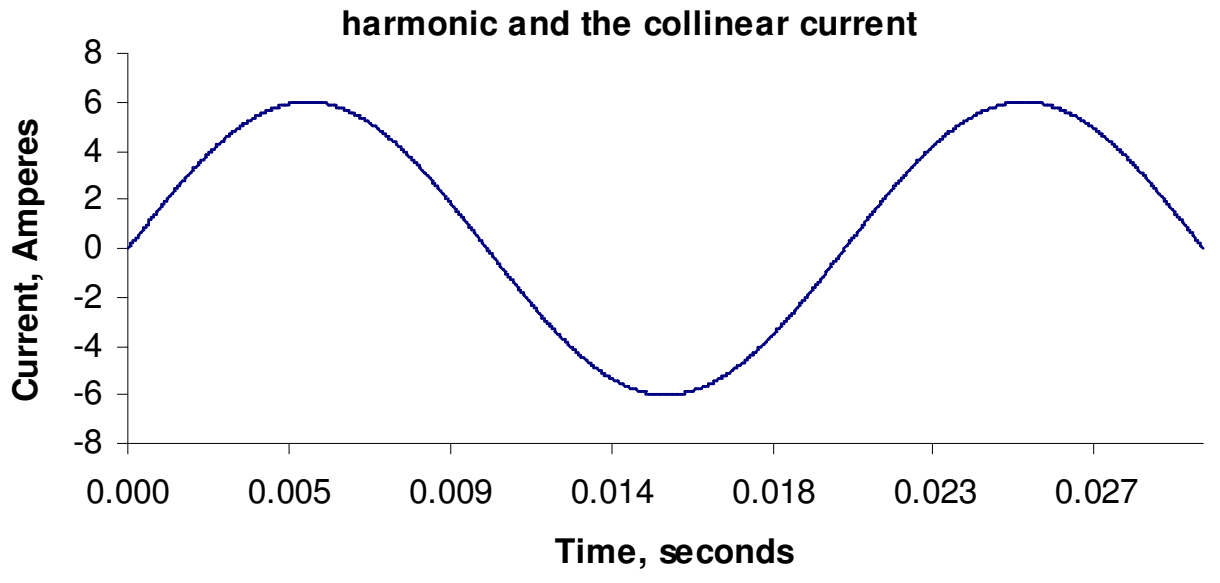

Fig. 3. Comparison of compensator control currents obtained by different calculation methods

\section{Results and discussion}

Based on the results of comparison of the two modes of compensator operation, it can be concluded that the amplitudes of compared currents differ by more than 10 percent. In the case of controlling the current compensator for the main harmonic of the load current (prototype), the compensator will be overloaded by the same amount of power, provided the mains voltage remains unchanged for both compared cases.

\section{Conclusions}

The algorithm of the compensator control system, based on the representation of the time functions of voltages and currents through vectors the coordinates of which are the values of time, and the projections are instantaneous values of currents and voltages allow to obtain the most informative parameters of the control system compensating device from the point of view of the optimitization of energy processes and minimization of energy losses in the system.

Practical application of the proposed calculation technique of decomposition of the instantaneous power into components allows in real time to improve the electromagnetic compatibility of loads with different types of current-voltage characteristics, to reduce the level of higher harmonics and network disturbances in power supply systems. [6]

The results of simulation modeling confirm the effectiveness of the proposed calculation method in comparison with the calculation procedure adopted for the prototype, due to the elimination overload of the compensating installation and the reduction of electric energy losses under equal operating conditions. 
Minimizing the losses of electricity with the wide use of modern power electronics devices that distort the forms of supply voltages and currents continues to be a serious scientific and technical task.

\section{Acknowledgements}

The research was supported by funding UDC 621.314.632.

\section{References}

1. Asanbaev Yu. A. Fundamentals of the energy processes theory in transformation plants: Dis. ... Dr. techn. Sciences: 05.09.12: St. Petersburg, 2012. - 285 c.

2. Demirchyan K.S. Reactive power for non-sinusoidal functions. Orthomality. Izvestiya of the Russian Academy of Sciences. Power engineering. 1992. № 1, pp. 15-38.

3. Demirchyan K.S. Decomposition of instantaneous power into constituents. - Izvestiya of the Russian Academy of Sciences. Power Engineering, 1994, No. 5, pp. 73-79.

4. Zharkov F.P. About one method of determining reactive power. // Izvestiya of the Academy of Sciences of the USSR. Energy and transport. 1984. № 2. pp. 73-81.

5. German-Galkin S.G. Virtual laboratories of semiconductor systems in Matlab-Simulink: Textbook. - St. Petersburg. : Publishing house "Lan", 2013. - 448 p .: ill.

6. Vorotnikov I.N., Mastepanenko, M.A. Kozmina, I.S. Algorithm for controlling the power factor correction device. Vorotnikov // Fedorov's Readings - 2015. XLV International Scientific and Practical Conference with Elements of a Scientific School (Moscow, November 11-13, 2015) / under total. Ed. B.I. Kudrin, Yu.V. Matyunina. - M .: Publishing house MEI. - 2015, pp 115-119. 